\title{
Selective decontamination of the digestive tract reduced intensive care unit and hospital mortality in adults
}

de Jonge E, Schultz MJ, Spanjaard L, et al. Effects of selective decontamination of digestive tract on mortality and acquisition of resistant bacteria in intensive care: a randomised controlled trial. Lancet 2003;362:1011-6.

\section{Does selective decontamination of the digestive tract (SDD) reduce mortality and acquisition of resistant bacteria in adult patients in the intensive care unit (ICU)?}

\section{METHODS}

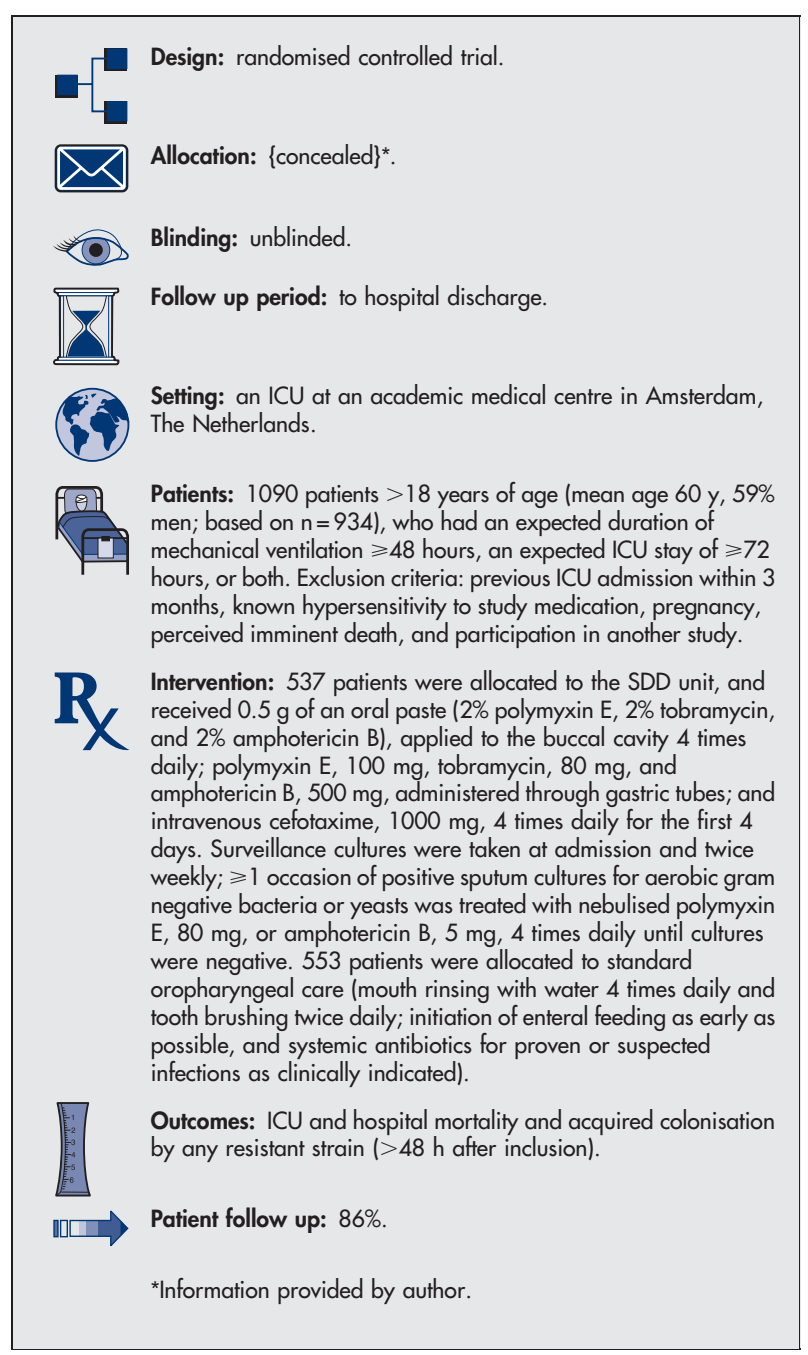

For correspondence: DrE Ede Jonge, Department of Intensive Care, Academic Medical Centre, University of Amsterdam, Amsterdam, Netherlands. e.dejonge@amc.uva.nl

Source of funding: not stated.

\section{MAIN RESULTS}

Patients in the SDD unit had lower ICU and hospital mortality rates than patients in the standard care unit (table). Follow up cultures were available for 773 patients (71\%). Patients in the SDD unit had lower rates of acquired colonisation with $\geqslant 1$ resistant strains of Pseudomonas aeruginosa or other gram negative bacteria than patients in the standard care unit (table).

\section{CONCLUSION}

Selective decontamination of the digestive tract reduced intensive care unit and hospital mortality among adult patients in the intensive care unit.

\section{Commentary}

DD selectively targets pathogenic organisms with the aim of reducing nosocomial infections. Although recent systematic reviews have shown reduced mortality associated with SDD, ${ }^{12}$ its use in ICUs has been challenged. In particular, concerns have been raised about the increased risk of resistance to antibiotics and the added costs of treating resistant nosocomial infections. ${ }^{3}$

The important work by De Jonge et al, however, suggests that SDD reduces resistant organism colonisation and increases patient survival. A strength of the study is the heterogeneous sample of patients allocated to 2 units in the same centre. The same medical staff treated patients with the same protocols in both units, except that one unit also used SDD. Although this was an open label (unblinded) study, the authors used objective outcome measures.

A similar percentage of patients in each group were lost to follow up, and the potential effects of these missing data on the overall findings are unknown. It is also possible that patterns of antibiotic resistance might have been detected if the study had continued. However, recruitment was stopped early because the units were being moved. Despite this, the authors have continued to monitor for antibiotic resistance in their unit, but have not found such a pattern.

ICU staff need to carefully consider SDD. Settings with lower baseline risks of nosocomial infection may not achieve the benefit reported by de Jonge et al. Although, de Jonge et al report the cost of antibiotic use for the 2 groups - with the results favouring the SDD group - a formal economic analysis is needed before the overall cost-benefit of SDD can be firmly established.

Bridie Kent, RN, PhD School of Nursing, University of Auckland Auckland, New Zealand

1 Nathens AB, Marshall JC. Arch Surg 1999;134:170-76.

2 D'Amico R, Pifferi S, Leonetti C, et al. BMJ 1998;316:1275-85.

3 Vincent JL. Lancet 2003;362:1006-7.

Selective decontamination of the digestive tract (SDD) $v$ standard oropharyngeal care for adult patients in the intensive care unit $(\mathrm{ICU})^{*}$

\begin{tabular}{lllll}
\hline Outcomes at discharge & SDD & Standard care & RRR (95\% CI) & NNT (CI) \\
\hline ICU mortality & $15 \%$ & $23 \%$ & $35 \%(15$ to 51$)$ & $13(8$ to 33$)$ \\
Hospital mortality & $24 \%$ & $31 \%$ & $22 \%(4$ to 37$)$ & $15(8$ to 83$)$ \\
Acquired colonisation with $\geqslant 1$ resistant & $16 \%$ & $26 \%$ & $38 \%(18$ to 54$)$ & $10(7$ to 23$)$ \\
strain of gram negative bacteriat & & & &
\end{tabular}

*Abbreviations defined in glossary; RRR, NNT, and Cl calculated from data in article.

†Based on data from 773 of 934 patients. 